\title{
The Theoretical Estimation of the Bioluminescent Efficiency of the Firefly via a Nonadiabatic Molecular Dynamics Simulation
}

\author{
Ling Yue, ${ }^{\dagger}$ Zhenggang Lan, ${ }^{* \dagger}$ and Ya-Jun Liu* ${ }^{\dagger}$ \\ ${ }^{\dagger}$ Key Laboratory of Theoretical and Computational Photochemistry, Ministry of Education, College of Chemistry, Beijing Normal \\ University, Beijing 100875, China \\ ${ }^{\ddagger}$ CAS Key Lab of Bio-based Materials, Qingdao Institute of Bioenergy and Bioprocess Technology, Chinese Academy of Sciences, \\ Qingdao, 266101, P.R. China
}

\section{Supporting Information}

ABSTRACT: The firefly is famous for its high bioluminescent efficiency, which has attracted both scientific and public attention. The chemical origin of firefly bioluminescence is the thermolysis of the firefly dioxetanone anion $\left(\mathrm{FDO}^{-}\right)$. Although considerable theoretical research has been conducted, and several mechanisms were proposed to elucidate the high efficiency of the chemi- and bioluminescence of $\mathbf{F D O}^{-}$, there is a lack of direct experimental and theoretical evidence. For the first time, we performed a nonadiabatic molecular dynamics simulation on the chemiluminescent decomposition of $\mathrm{FDO}^{-}$under the framework of the trajectory surface hopping ( $\mathrm{TSH}$ ) method and theoretically estimated the chemiluminescent quantum yield. The TSH simulation reproduced the gradually reversible charge-transfer initiated luminescence mechanism proposed in our previous study. More importantly, the current study, for

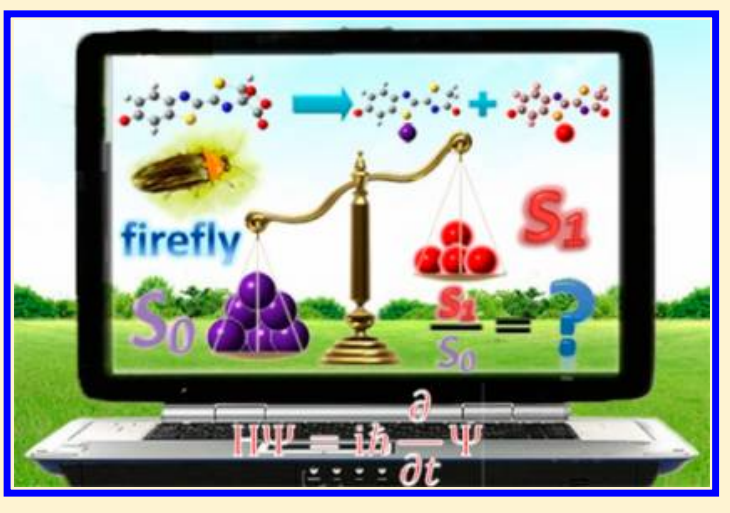
the first time, predicted the bioluminescence efficiency of the firefly from a theoretical viewpoint, and the theoretical prediction efficiency is in good agreement with experimental measurements.

$\mathrm{L}$ iving organisms that can produce cold light are extremely fascinating, and accordingly, great efforts have been devoted to elucidating the mechanism behind such mysterious light over several centuries. This light emission, denoted as "bioluminescence", is a special type of chemiluminescence that is the consequence of chemical reactions in living organisms.

The firefly may be the most famous and important bioluminescence system. Due to their extremely high luminescent efficiency $(41.0+7.4 \%){ }^{1}$ the chemical origin of their luminescence has received great interest from experimental and theoretical scientists. These studies determined that the chemical conversion from D-luciferin to oxyluciferin is responsible for the bioluminescence emission of the firefly, as is shown in Scheme 1. The D-luciferin first reacts with ATP in the presence of a catalytic enzyme (luciferase) and cofactors $\left(\mathrm{Mg}^{2+}\right)$ to form an intermediate (luciferin-AMP) that can be oxidized by $\mathrm{O}_{2}$ to produce the so-called "firefly dioxetanone" (FDO). Due to low stability, FDO quickly decomposes into oxyluciferin and $\mathrm{CO}_{2}$. During this thermolysis step, a portion of the oxyluciferin is nonradiatively excited to its first singlet excited state $\left(S_{1}\right)$. In other words, the ground to excited state $\left(S_{0} \rightarrow S_{1}\right)$ nonadiabatic transition is induced by a chemical decomposition reaction, and the process is thus called chemiexcitation. Finally, the decay of the excited-state oxyluciferin to its $S_{0}$ state emits the visible light. ${ }^{2}$

It is generally believed that the decomposition of FDO starts from its anionic form $\left(\mathrm{FDO}^{-}\right)$, rather than its neutral form
(FDOH $)^{2}$ Both experimental ${ }^{3-5}$ and theoretical ${ }^{6-10}$ studies have indicated that firefly bioluminescence corresponds to the emission from the $S_{1}$-state anionic oxyluciferin $\left(\mathrm{OxyLH}^{-}\right)$ instead of the neutral one $\left(\mathrm{OxyLH}_{2}\right)$. This provides strong evidence on the dominant role of $\mathrm{FDO}^{-}$because its decomposition can directly produce the $S_{1}$ state of $\mathrm{OxyLH}^{-}$. The study of biomimic 1,2-dioxetane systems also provides additional support for the importance of $\mathbf{F D O}^{-}$. As McCapra et al. ${ }^{11}$ and Zaklik et al. ${ }^{12}$ note, a charge-transfer (CT) or electron transfer (ET) process from $\mathrm{OxyLH}_{2} / \mathrm{OxyLH}^{-}$to a $\mathrm{CO}_{2}$ moiety plays a very important role in the production of chemi- and bioluminescence with high efficiency. ${ }^{11,13-16}$ Nevertheless, it is clear that the $\mathrm{CT} / \mathrm{ET}$ process is considerably more feasible in FDO $^{-}$than in FDOH.

The chemically initiated electron exchange luminescence (CIEEL) proposed in the $1970 \mathrm{~s}^{13,14,17,18}$ is widely accepted as the mechanism for the chemiluminescence of 1,2-dioxetane and its derivatives, including firefly bioluminescence. The CIEEL mechanism assumes that the thermolysis of 1,2-dioxetane derivatives is initiated by a full single electron transfer (ET) from the electron donor (e.g., from the oxyanion on the benzothiazole group in $\mathbf{F D O}^{-}$to the peroxide bond), and the chemi-excitation takes place during the next step of the back

Received: October 30, 2014

Accepted: January 23, 2015

Published: January 23, 2015 
Scheme 1. Rough Mechanism of Firefly Bioluminescence

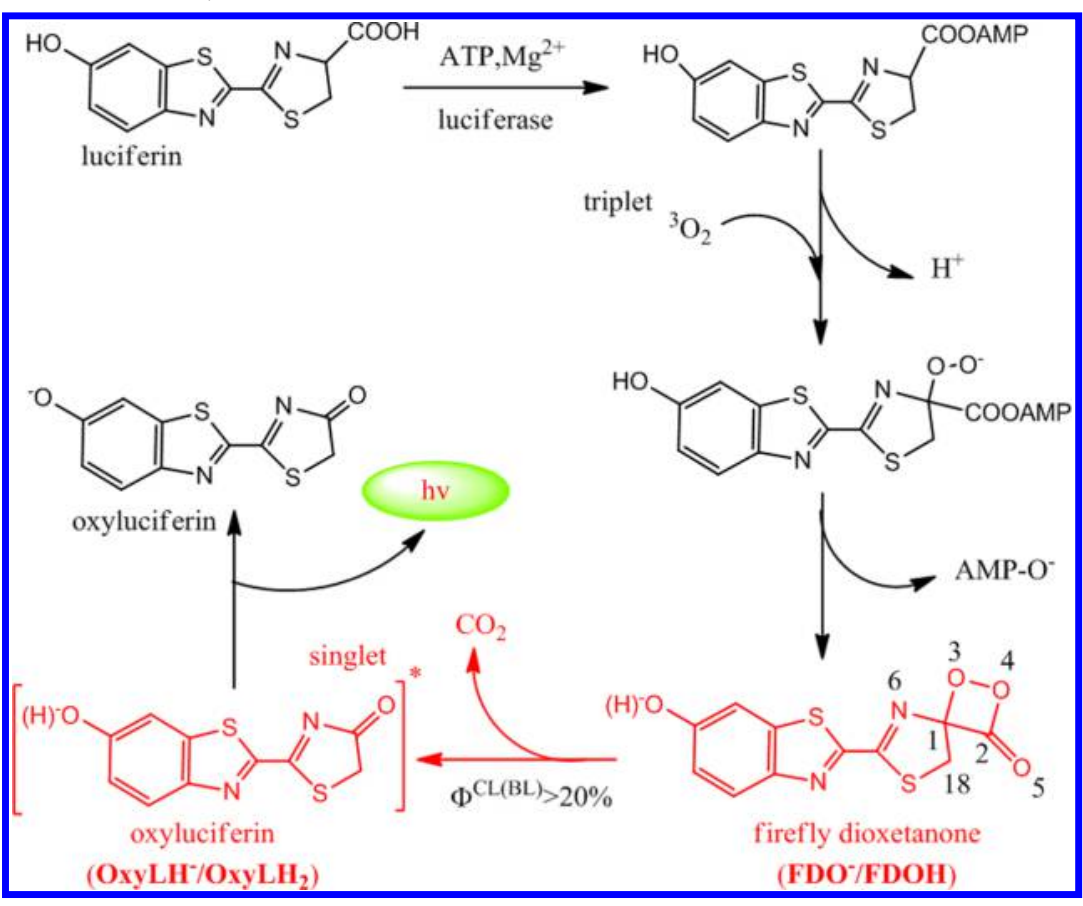

electron transfer (BET). However, the existence of a full single $\mathrm{ET} / \mathrm{BET}$ process and the efficiency of the BET excitation were controversial. Other mechanisms such as charge transfer induced luminescence (CTIL) $)^{19-21}$ and charge transfer induced decomposition (CTID $)^{22-25}$ were also proposed to elucidate the anionic decomposition of 1,2-dioxetanes, the formation of the $S_{1}$ state product, and the high luminescent efficiency. The CTIL mechanism assumes that a partial CT process from $\mathrm{OxyLH}^{-}$to $\mathrm{CO}_{2}$, rather than a full ET one, induces the decomposition of $\mathbf{F D O}^{-}$, and the excited-state product is produced by nonadiabatic transitions in the vicinity of a conical intersection (CI) instead of the $\mathrm{CO}_{2} \rightarrow \mathrm{OxyLH}^{-}$ BET process. Our previous theoretical investigation using stateof-the-art multiconfiguration calculations ${ }^{26}$ also supported the CT-induced (anionic) decomposition. It showed that the chemiluminescent decomposition of $\mathbf{F D O}^{-}$can be described by the gradually reversible charge transfer initiated luminescence (GRCTIL) mechanism, which consists of a gradual CT and back charge transfer (BCT) process accompanied by the cleavages of $\mathrm{O}-\mathrm{O}$ and $\mathrm{C}-\mathrm{C}$ bonds, respectively. During the $\mathrm{CT} / \mathrm{BCT}$ process, the "double crossing" (two CIs) between the $S_{0}$ and $S_{1}$ potential energy surfaces (PESs) along the reaction pathway is responsible for the high chemi-excitation efficiency. Moreover, the GRCTIL mechanism also explains some experimental observations on the chemiluminescence of 1,2dioxetane derivatives successfully. ${ }^{23}$

Up to the present, all of these studies and proposed mechanisms were based on either experimental assumptions by indirect measurements or theoretical computations by characterizing the key points (equilibriums, intermediates, transition states (TSs) and CIs) and PESs. They could not directly verify the proposed mechanisms or provide the corresponding luminescent efficiencies.

Recently, Farahani et al. ${ }^{27}$ studied the dynamics of 1,2dioxetane, which is the simplest analogue of FDO, by high-level multireference calculation and ab initio molecular dynamics (AIMD) simulation. Their results provide further details on the mechanisms that qualitatively explain the high ratio of triplet to singlet dissociation products. However, the AIMD simulation is based on the Born-Oppenheimer approximations and does not include nonadiabatic transitions, so it could not produce the quantum yield of the excited-state product. Thus, this approach does not provide a complete description of the formation of the excited-state product, the light emitter, which is essentially responsible for chemi- and bioluminescence. Moreover, this study focused on the decomposition of the neutral FDO analogue, not on the decomposition of $\mathrm{FDO}^{-}$. The decomposition mechanisms of $\mathrm{FDOH}$ and $\mathrm{FDO}^{-}$are quite different. ${ }^{19,26,28-33}$

Therefore, neither experimental nor theoretical studies have thus far provided direct evidence on the roles of the CT process and nonadiabatic transitions in the $\mathbf{F D O}^{-}$decomposition. The study of the formation probability of $\mathrm{S}_{1}$ state $\mathrm{OxyLH}^{-}$, which is essential to estimating the chemi- or bioluminescent efficiency for 1,2-dioxetane and its derivatives, is also lacking.

The chemi- or bioluminescent efficiency $\left(\Phi^{\mathrm{CL}}\right.$ or $\left.\Phi^{\mathrm{BL}}\right)$ is defined as the quantum yield of the production of a photon from a single reactant. ${ }^{34}$ It can be described as the product of the quantum yields of two independent processes, (i) the formation of electronic excited-state products during the thermolysis of FDO (chemi-excitation process), i.e., the yield of the chemi-excited state product $\left(\Phi^{\mathrm{CS}}\right)$, and (ii) the radiative decay of the excited product (fluorescence process), i.e., the fluorescent yield $\left(\Phi^{\mathrm{FL}}\right)$. Because step (i) directly determines the yield of the light emitter and further influences the luminescent efficiency of the firefly, it has been widely investigated experimentally $^{14,35}$ and theoretically. ${ }^{19,20,29,31,36}$ Thus, theoretically computing the quantum yield of chemiexcitation $\left(\Phi^{C S}\right)$ for the proposed mechanisms, which is the branch ratio of the $S_{0^{-}}$and $S_{1}$-state products after the nonadiabatic transitions, can offer direct and powerful evidence to clarify the actual decomposition process that produces the light. 
As many 1,2-dioxetane derivatives are successfully applied in chemical analysis and clinical assays, ${ }^{37-43}$ a detailed understanding of the chemical mechanism governing the production of chemi- and bioluminescence is extremely important. Thus, for the first time, we performed a nonadiabatic molecular dynamics simulation on the decomposition process of FDO $^{-}$, the most representative derivative of 1,2-dioxetane. Because the current dynamical calculations include the nonadiabatic transition between the $S_{0}$ and $S_{1}$ states, it is possible to estimate the quantum yield of the $S_{1}$-state $\mathrm{OxyLH}^{-}\left(\Phi^{\mathrm{CS}}\right)$ and furthermore of the chemiluminescence $\left(\Phi^{\mathrm{CL}}\right)$. The simulation results also provide direct evidence to clarify the role of the $\mathrm{CT}$ process in the decomposition reaction and identify the relevant nuclear motions. This work should provide more detailed information to understand the mystery of the bioluminescence of the firefly.

Review of Previous Study. In previous work, we constructed the reaction pathways of FDO in both its neutral (FDOH) and anionic $\left(\right.$ FDO $\left.^{-}\right)$forms at both the CASPT2 and DFT/TDDFT levels. The Coulomb-attenuated hybrid exchange-correlation (CAM-B3LYP) functional was used in the DFT/TDDFT calculation. ${ }^{26}$ The decomposition of FDOH requires a two-step pathway via two TSs (Figure 1 and Figure S2B). The two TSs

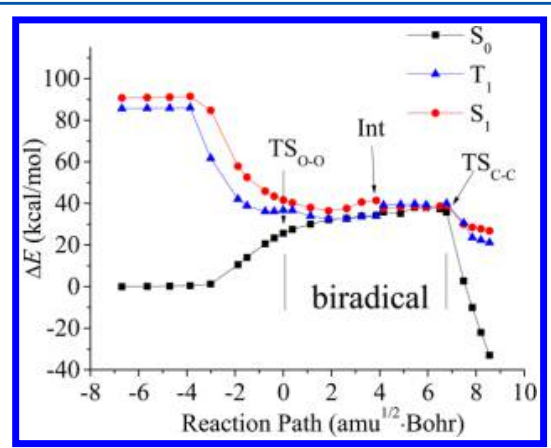

Figure 1. $S_{0}, T_{1}$, and $S_{1}$ PESs for the decompositions of FDOH, calculated at the CASPT2//CAM-B3LYP level (Combination of Figure 2A and Figure 4 in ref 26, adapted with permission from ref 26. Copyright 2012 American Chemical Society).

correspond to the stretching of the $\mathrm{O}_{3}-\mathrm{O}_{4}$ and $\mathrm{C}_{1}-\mathrm{C}_{2}$ bonds, labeled as $\mathrm{TS}_{\mathrm{O}-\mathrm{O}}$ and $\mathrm{TS}_{\mathrm{C}-\mathrm{C}}$, respectively. The ground and excited $\left(T_{1}\right.$ and $\left.S_{1}\right)$ states have no obvious CT properties. They become close to each other and become nearly degenerate at a flat biradical region. The nearly degenerate non-CT PESs leads to nonadiabatic transitions, which provide the possibility for $S_{0}$ $\rightarrow \mathrm{T}_{1}$ intersystem crossing. ${ }^{26}$ This mechanism is similar to 1,2 dioxetanone, ${ }^{30}$ whose thermolysis will produce a large quantity of triplet products without fluorescent activity. Thus, the decomposition of FDOH is not expected to provide a high quantum yield of chemiluminescence. The previous work basically rules out the possible involvement of FDOH, so this species is not important in our next study.

The decomposition of the $\mathrm{FDO}^{-}$species follows a completely different mechanism because it takes place through an asynchronous two-stage pathway via only one CT TS $\left(\mathrm{TS}_{\mathrm{O}-\mathrm{O}}\right)$ (Figure 2 and Figure S2E). Neither the radical anion pair nor the full single ET/BET required by the CIEEL mechanism was observed in the thermolysis of $\mathrm{FDO}^{-}$. Thus, we proposed an accurate mechanism denoted as gradually reversible CT-initiated luminescence (GRCTIL) (see ref 26 for details). Instead of the nearly degenerate ground and excited

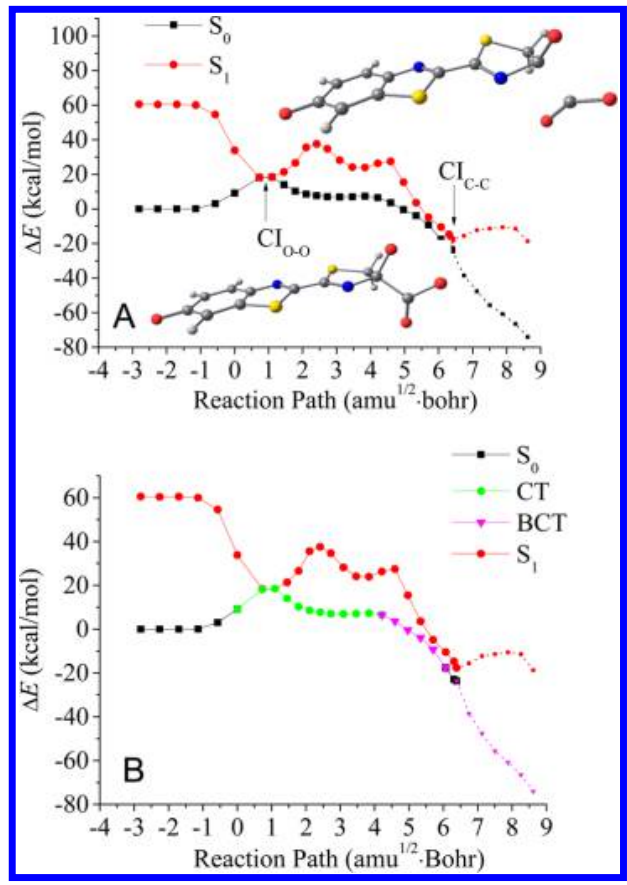

Figure 2. $S_{0}$ and $S_{1}$ PESs (A, modified from Figure $2 D$ of ref 26, adapted with permission from ref 26. Copyright 2012 American Chemical Society), CT and BCT on $\mathrm{S}_{0}$ PES (B, reproduced from Figure 3 of ref 26, reprinted with permission from ref 26. Copyright 2012 American Chemical Society) for the decompositions of FDO $^{-}$ calculated at the CASPT2//CAM-B3LYP level.

PESs in the biradical region along the FDOH decomposition pathway, "double crossings" (two CIs) between the $S_{0}$ and $S_{1}$ PESs (Figure 2) presented along the pathway of the decomposition of $\mathbf{F D O}^{-}{ }^{26,36}$ Moreover, different from the neutral (FDOH) decomposition, the near degenerated $S_{0}$ and $\mathrm{T}_{1}$ PESs of FDO $^{-}$at the biradical region do not fulfill the ElSayed's rule ${ }^{44}$ for the $S_{0} \rightarrow T_{1}$ transition to form the triplet products, which is forbidden in the zero order. The high yield of bioluminescence for the firefly was assumed to be relevant to the $S_{0} \rightarrow S_{1}$ nonadiabatic transition in the vicinity of the two CIs.

Validation of the SF-BHHLYP Approach. The reliability of SFBHHLYP method used in current work needs to be examined by comparing the results obtained from the previous (TD)CAM-B3LYP and multiconfigurational study. ${ }^{26}$ As mentioned in previously, theoretical calculations by the (TD-)CAMB3LYP and CASPT2 methods show that the decomposition of FDO $^{-}$is governed by a CT-induced asynchronous concerted process, which is completely different from the neutral form's decomposition. $^{26}$ In fact, the SF-BHHLYP/6-31G and SF$\mathrm{BHHLYP} / 6-31 \mathrm{G}^{* *}$ calculations in the current work reproduce the correct mechanism very well according to the following comparisons.

First, a unique TS in the reaction pathway for the decomposition of FDO $^{-}$was located at the SF-BHHLYP/6$31 \mathrm{G}$ and SF-BHHLYP/6-31G** levels, consistent with the results at the (TD-)CAM-B3LYP/6-31G** level in the previous study. ${ }^{26}$ The imaginary vibrational mode shows that the TS is characterized by the stretching of $\mathrm{O}_{3}-\mathrm{O}_{4}$. According to the key geometric parameters at both the ground-state minimum (Min) and the TS (Table S1), the geometric distortion from Min to TS in the current calculation is very similar to the previous results at the CAM-B3LYP/6-31G** 


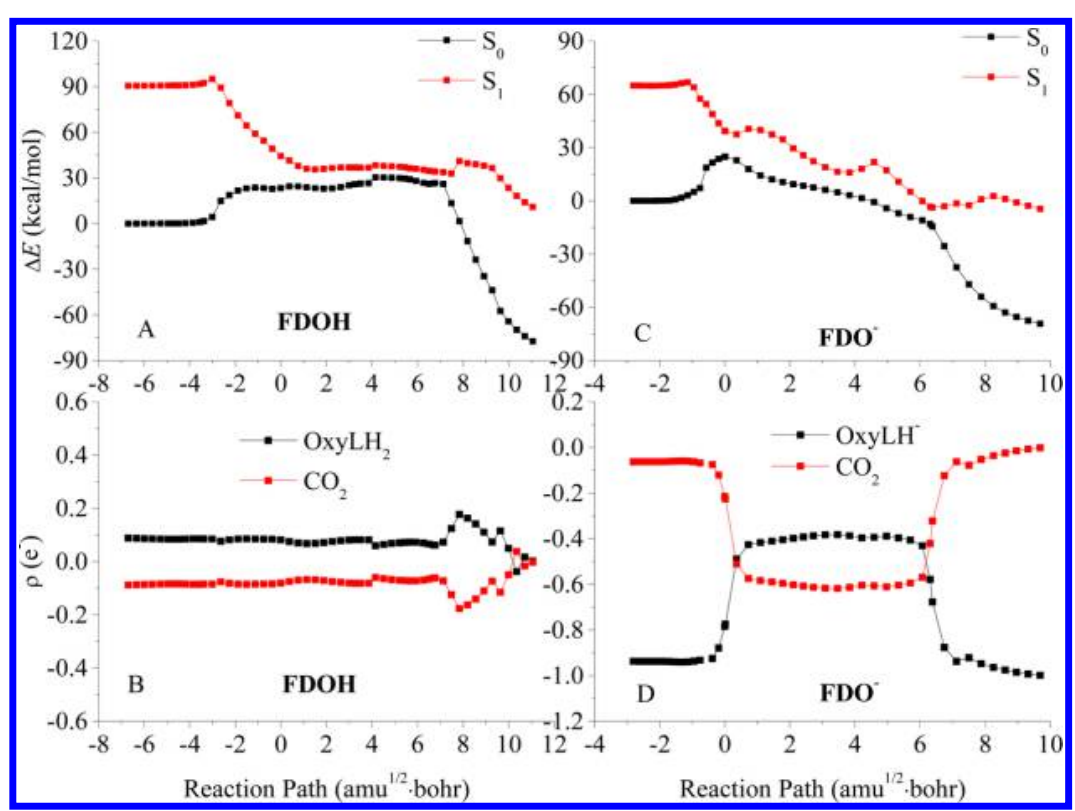

Figure 3. $S_{0}$ and $S_{1}$ PES and the Mulliken charge population of $S_{0}$ PES, calculated at the SF-BHHLYP/6-31G** level (current work). The reaction pathway is based on the IRC of previous calculations at the CAM-B3LYP/6-31G** level (data from Figure S11 A and C of ref 26).

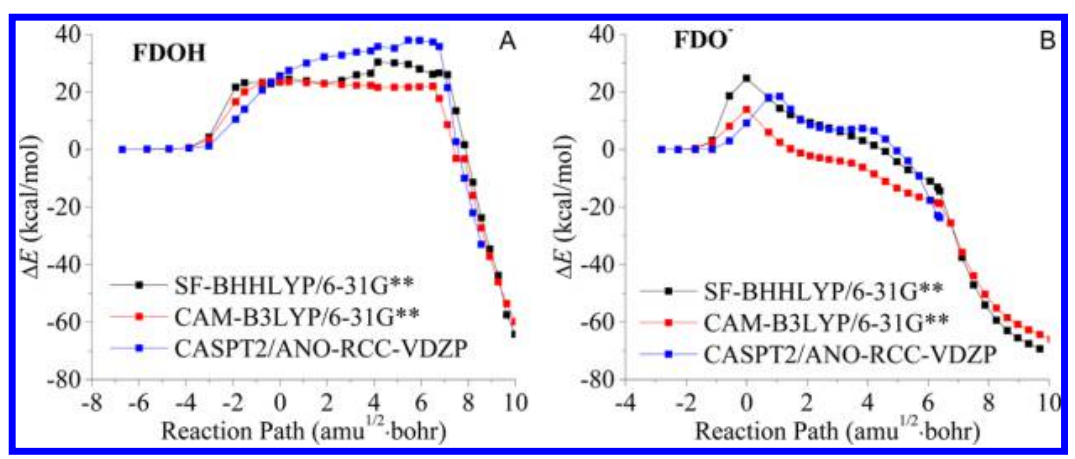

Figure 4. Comparison of $S_{0}$ PES, calculated at the SF-BHHLYP/6-31G** (current work), CAM-B3LYP/6-31G**and CASPT2/ANO-RCC-VDZP (data from Figure S12 of ref 26) levels. The reaction pathway is based on the IRC of previous calculations at the CAM-B3LYP/6-31G** level.

level. Second, the ground $\left(S_{0}\right)$ and excited $\left(S_{1}\right)$ state PESs calculated at the SF-BHHLYP/6-31G** (Figure $3 \mathrm{~A}$ and $\mathrm{C}$ ) and SF-BHHLYP/6-31G (Figure S1A and C) levels show the similar tendency as the (TD-)CAM-B3LYP (Figure S2A and D) and CASPT2 (Figure 1, 2A, S3A, and C) levels in the previous work. Third, the Mulliken charge population at the SF-BHHLYP/6-31G** (Figure 3B and D) and SF-BHHLYP/ 6-31G (Figure S1B and D) levels show a non-CT thermolysis for FDOH and a gradually reversible CT (CT/BCT) decomposition for FDO $^{-}$. The $\mathrm{CT}$ and BCT between the $\mathrm{OxyLH}^{-}$and $\mathrm{CO}_{2}$ moieties in $\mathbf{F D O}^{-}$also take place during the $\mathrm{O}_{3}-\mathrm{O}_{4}$ and $\mathrm{C}_{1}-\mathrm{C}_{2}$ bond breaking processes, respectively, consistent with the results obtained at the CAM-B3LYP (Figure S2C and F) and CASPT2 (Figure S3B and D) levels.

Overall, the decomposition mechanism by the SF-BHHLYP calculations are qualitatively consistent with those using the (TD-)CAM-B3LYP and CASPT2 methods. Particularly, the mechanism of the dominant reaction channel $\left(\right.$ FDO $^{-}$ decomposition) seems to be well-reproduced by the SFBHHLYP method (Figure 4B and S4B). Although some minor differences may still exist for the decomposition path of the neutral species FDOH (Figure 4A and S4A), this reaction channel does not play an important role according to previous studies. In addition, the minor difference between the different methods is acceptable, as one of our aims in this work is to estimate the magnitude of the quantum yield of the species with strong emission during the decomposition of $\mathbf{F D O}^{-}$and the resulted chemiluminescence efficiency. As it is still impossible to perform nonadiabatic dynamics simulations for such complex systems at the CASPT2 level, the SF-BHHLYP method is a proper choice for the trajectory surface hopping (TSH) simulation of the FDO $^{-}$decomposition process to achieve a balance between accuracy and cost.

Decomposition Dynamics of $\mathbf{F D O}^{-}$. The dynamics of the FDO $^{-}$decomposition was investigated by the TSH simulation at both the SF-BHHLYP/6-31G and SF-BHHLYP/6-31G** levels. $^{26}$

Asynchronous Decomposition. The geometric distributions of $\mathrm{C}_{1}-\mathrm{C}_{2}$ and $\mathrm{O}_{3}-\mathrm{O}_{4}$ bond lengths at different times (Figure 5 and more details in Figure S5) clearly indicate the system evolution with time. In the early stage of the reaction (within $30 \mathrm{fs}$ ), only fast $\mathrm{O}_{3}-\mathrm{O}_{4}$ elongation takes place, while the $\mathrm{C}_{1}-$ $\mathrm{C}_{2}$ distance remains unchanged until the $\mathrm{O}_{3}-\mathrm{O}_{4}$ distance reaches $\sim 3.0 \AA$. After $50 \mathrm{fs}$, the further cleavage of $\mathrm{C}_{1}-\mathrm{C}_{2}$ bond is also observed. Therefore, both the distribution of bond lengths and the variation of average values (Figure S6 and S7) show the mechanism of the asynchronous $\mathrm{O}_{3}-\mathrm{O}_{4}$ and $\mathrm{C}_{1}-\mathrm{C}_{2}$ cleavage taking place at $0-30 \mathrm{fs}$ and $>30 \mathrm{fs}$, respectively. 


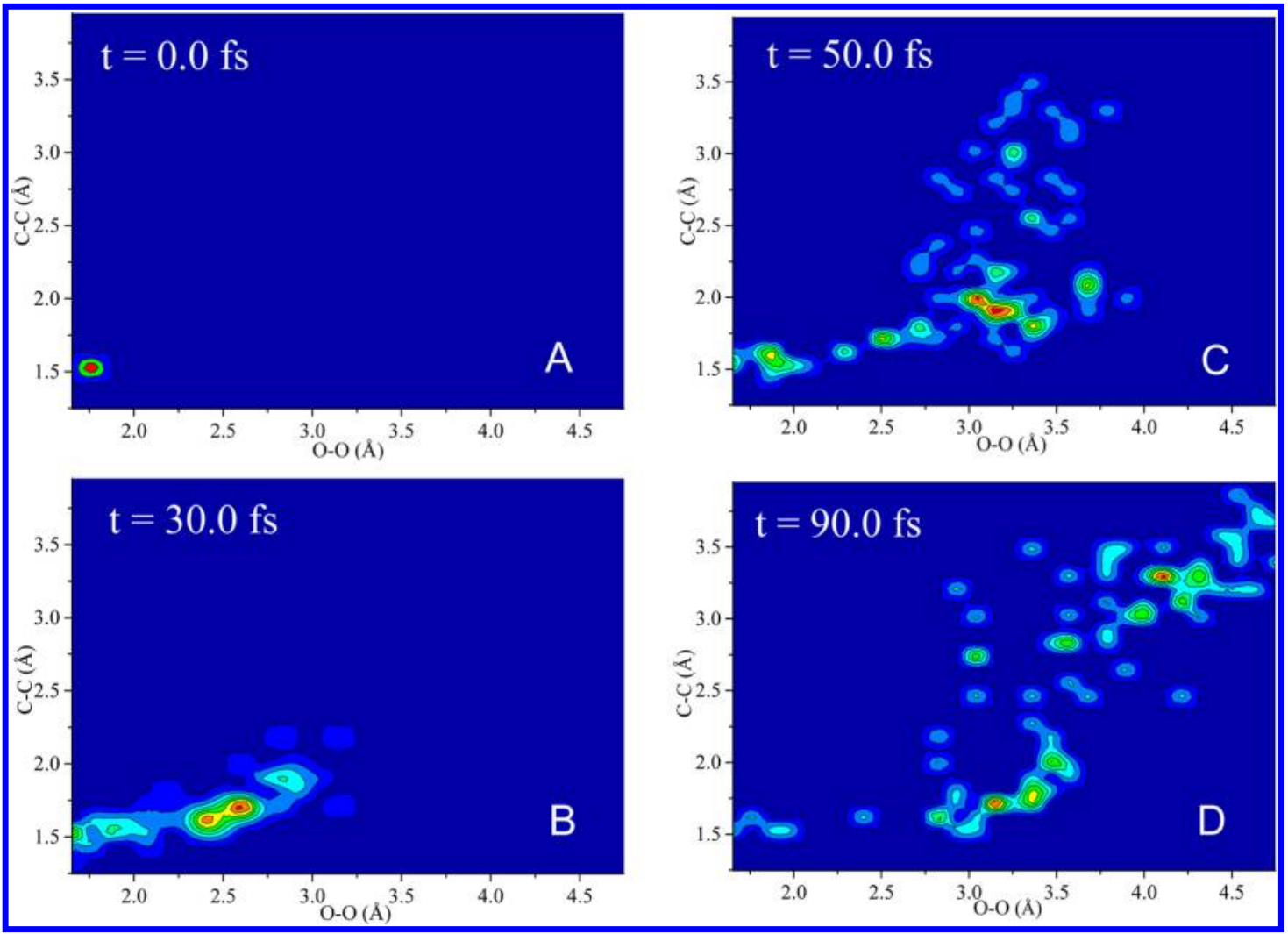

Figure 5. Two-dimensional distribution of the $\mathrm{C}_{1}-\mathrm{C}_{2}$ and $\mathrm{O}_{3}-\mathrm{O}_{4}$ bond lengths at $0,30,50$, and 90 fs during the dynamics evolution, obtained using the TSH dynamics at the SF-BHHLYP/6-31G** level.

Therefore, the TSH dynamics provides a clear view of the mechanism of a two-stage decay process, consistent with previous studies. ${ }^{26,27}$

Gradual CT and BCT. The evolution of the average charge population (Figure 6 and S8) clearly displays a gradual negative

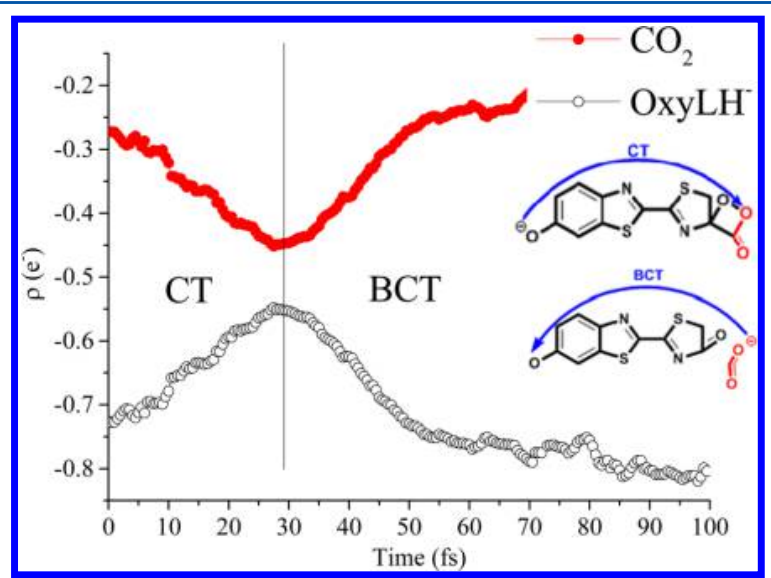

Figure 6. Time evolution of the average Mulliken charge population of oxyluciferin and the $\mathrm{CO}_{2}$ moiety during the dynamics evolution of FDO $^{-}$(average over 100 trajectories), obtained by the TSH dynamics simulation at the SF-BHHLYP/6-31G** level.

$\mathrm{OxyLH}^{-} \rightarrow \mathrm{CO}_{2} \mathrm{CT}$ within $\sim 30$ fs and a gradual $\mathrm{CO}_{2} \rightarrow$ $\mathrm{OxyLH}^{-}$BCT at 30-70 fs. The time scales of the CT and BCT match exactly with the asynchronous cleavage of the $\mathrm{O}_{3}-\mathrm{O}_{4}$ $(0-30 \mathrm{fs})$ and $\mathrm{C}_{1}-\mathrm{C}_{2}$ (>30 fs) bonds (Figure 5), respectively. This shows that the so-called GRCTIL mechanism is driven by the successive cleavage of the $\mathrm{O}_{3}-\mathrm{O}_{4}$ and $\mathrm{C}_{1}-\mathrm{C}_{2}$ bonds.
Hence, the result from the TSH simulation commendably reproduces the decomposition of $\mathrm{FDO}^{-}$and confirms the previous gradual $\mathrm{CT} / \mathrm{BCT}$ mechanism derived from reaction pathway calculations by the SF-BHHLYP, CAM-B3LYP, and CASPT2 methods. $^{26}$

Role of Two CIs in Dynamics. The time evolution of the average fractional occupations of the $S_{0}$ and $S_{1}$ states (Figures 7 and S9) shows that the dynamical process can be separated into two stages. In the first stage (stage 1 in Figures 7 and S9), the average fractional occupation of the $S_{1}$ state increases from zero to approximately 0.3 , implying the formation of the excited state by chemi-excitation within 30 fs. During this period, the

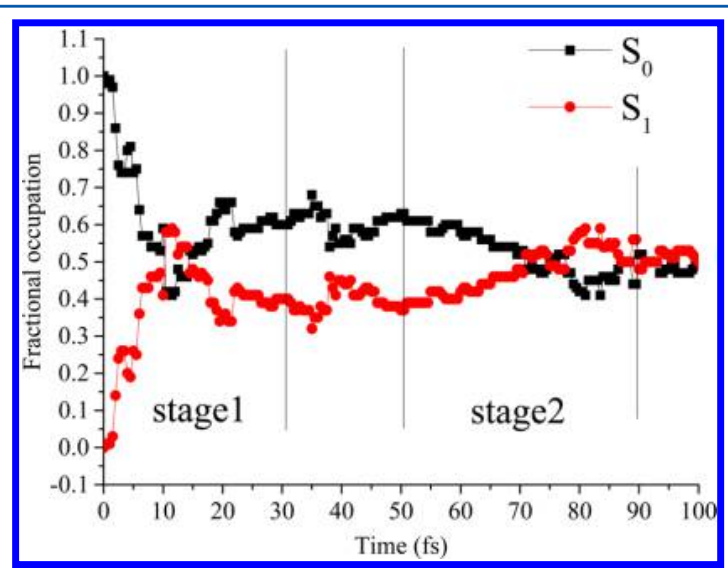

Figure 7. Time evolution of the fractional occupation of the $S_{0}$ and $S_{1}$ states during the dynamics evolution of FDO $^{-}$(average over 100 trajectories), obtained by the TSH dynamics simulation at the SFBHHLYP/6-31G** level. 
$\mathrm{O}_{3}-\mathrm{O}_{4}$ bond experiences a clear prolongation (up to 1.8-3.0 $\AA$, Figure $5 \mathrm{~B}$ ), while the $\mathrm{C}_{1}-\mathrm{C}_{2}$ bond only becomes slightly longer (1.5-1.7 $\AA$, Figure 5B). At the same time, the pronounced $\mathrm{OxyLH}^{-} \rightarrow \mathrm{CO}_{2} \mathrm{CT}$ is also observed (Figures 6 and S8), implying that the initial reaction is governed by the $\mathrm{CI}_{\mathrm{O}-\mathrm{O}}$. In the second stage (stage 2 in Figures 7 and S9), the average fractional occupation of the $S_{1}$ state increases slightly from $30 \mathrm{fs}$ and becomes stable after $90 \mathrm{fs}$. During this stage, the significant prolongation of the $\mathrm{C}_{1}-\mathrm{C}_{2}$ bond (2.0-2.6 $\AA$, Figure $5 \mathrm{C})$ induces the $\mathrm{CO}_{2} \rightarrow \mathrm{OxyLH}^{-} \mathrm{BCT}$, indicating that the $\mathrm{CI}_{\mathrm{C}-\mathrm{C}}{ }^{26}$ becomes dominant. Therefore, the two rising periods for the $S_{1}$ population could be attributed to two successive nonadiabatic transitions governed by the two CIs. Therefore, the results of the TSH calculations not only display the role of the two CIs in the decomposition dynamics of DDO $^{-}$but also primarily support the successive $\mathrm{CT}$ and $\mathrm{BCT}$ required by the GRCTIL mechanism. ${ }^{26}$

Quantum Yield of FDO $^{-}$Decomposition. At the SF-BHHLYP/ 6-31G** level, the TSH calculations indicate that FDO $^{-}$ decomposes completely at 150 fs (Figure S5 and S6). Sixtyone and 39 trajectories were found on the $S_{0}$ and $S_{1}$ surfaces, respectively. Thus, the theoretical quantum yield of chemiexcitation $\left(\Phi^{C S}\right)$ of $\mathbf{F D O}^{-}$in the gas phase is $39 \%$. This value falls into the range of experimental measurements $\left(\Phi^{\mathrm{CS}}=33 \%\right.$ $\sim 55 \%$ in different organic solvents) of the oxyluciferin analogues in which two $\alpha$-hydrogens of the carbonyl group were substituted by two methyl groups. ${ }^{45}$ Using the experimental fluorescent quantum yield $\left(\Phi^{\mathrm{FL}}=62 \%\right)$ of the oxyluciferin analogue, the overall chemiluminescent efficiency $\left(\Phi^{\mathrm{CL}}\right)$ in the $\mathbf{F D O}^{-}$decomposition in the gas phase may be approximately estimated to be $24 \%\left(\Phi^{\mathrm{CL}}=\Phi^{\mathrm{CS}} \times \Phi^{\mathrm{FL}}\right)$. Similar theoretical $\Phi^{\mathrm{CS}}(54 \%)$ and $\Phi^{\mathrm{CL}}(33 \%)$ values in the gas phase were obtained at the BHHLYP/6-31G level. Although the estimated chemiluminescent efficiencies (24\% and 33\%) are slightly lower than the real value of firefly luminescence $\left(\Phi^{\mathrm{BL}}=\right.$ $41 \%),{ }^{1}$ possibly due to the gas phase model, the current work excellently explains the highly efficient production of bioluminescence in the firefly. Moreover, according to the nonadiabatic dynamics features discussed above, two successive $\mathrm{S}_{0} / \mathrm{S}_{1}$ CIs play essential roles to control the formation of $\mathrm{OxyLH}^{-}$in its $\mathrm{S}_{1}$ state responsible for fluorescent emission. Thus, this "double-crossing" feature provides an interesting explanation for the high chemi- and bioluminescent efficiency of $\mathrm{FDO}^{-}$and other 1,2-dioxetane derivatives. However, a previous study ${ }^{36}$ by Morokuma's group only found the second CI, which is significantly different from this study and our previous studies. $^{26,29,30,32}$

The current work represents the first effort to simulate the nonadiabatic dynamics of the firefly $\mathrm{FDO}^{-}$and estimate the quantum yield of the fluorescence emitted from the $S_{1}$ state generated by chemi-excitation. The decomposition dynamics of FDO $^{-}$were investigated by the TSH method with the SF-DFT calculations. At first, we reinvestigated the decomposition mechanism of FDO $^{-}$by the SF-DFT method for validation. The results of the SF-DFT approach are consistent with previous state-of-the-art multiconfigurational computations. The TSH nonadiabatic dynamics simulation fits well with previous CASPT2//CAM-B3LYP results. The geometric distributions of the $\mathrm{O}_{3}-\mathrm{O}_{4}$ and $\mathrm{C}_{1}-\mathrm{C}_{2}$ distances at different times clearly show a successive cleavage of the two bonds and imply an asynchronous decomposition. Corresponding to the bond breaking of $\mathrm{O}_{3}-\mathrm{O}_{4}$ and $\mathrm{C}_{1}-\mathrm{C}_{2}$, the evolution of the average charge population also clearly displays a gradual negative CT $\left(\mathrm{OxyLH}^{-} \rightarrow \mathrm{CO}_{2}\right)$ and BCT $\left(\mathrm{CO}_{2} \rightarrow \mathrm{OxyLH}^{-}\right)$, respectively. In addition, the evolution of the average fractional occupation of the $S_{1}$ state also displays the important population transfer twice. Their time scales are coincident with the cleavage of the $\mathrm{O}_{3}-\mathrm{O}_{4}$ and $\mathrm{C}_{1}-\mathrm{C}_{2}$ bonds, also implying the existence of two successive $S_{0} / S_{1}$ CIs responsible for the nonadiabatic transitions producing $\mathrm{OxyLH}^{-}$in the $\mathrm{S}_{1}$ state. The non-negligible yields of the emission species $\left(\Phi^{\mathrm{CS}}=\right.$ $39 \%)$ and luminescence $\left(\Phi^{\mathrm{CL}}=24 \%\right)$ derived by the nonadiabatic dynamics of $\mathbf{F D O}^{-}$demonstrate that the two $\mathrm{S}_{0} / \mathrm{S}_{1}$ CIs in anionic decomposition probably govern the high chemi- and bioluminescent efficiency of firefly dioxetanone and its analogues. Therefore, the current work not only offers direct evidence to support the dominant role of anionic decomposition but also validates the GRCTIL mechanism.

\section{COMPUTATIONAL DETAILS}

The chemiluminescent decomposition of FDO $^{-}$was investigated by on-the-fly TSH dynamics ${ }^{46,47}$ in the framework of Tully's fewest switches approach. It is well-known that Tully's $\mathrm{TSH}$ approach suffers from the deficiencies in the description of the dual crossing on the potential-energy surface, ${ }^{48}$ our current treatment (even with decoherent correction ${ }^{49,50}$ ) is rather approximated. However, since we only focus on the qualitative understanding of nonadiabatic dynamics, the involved nuclear motions and chemiluminescence, Tully's TSH approach was employed to treat such medium-sized molecule due to its simplicity. Dynamics treatment with other more accurate theoretical methods should represent the great challenge for the future. The potential energies and nuclear gradients were computed by the linear-response time-dependent density functional theory (LR-TDDFT). The spin-flip (SF) scheme $e^{51-54}$ within the Tamm/Dancoff approximation (TDA $)^{55-57}$ was employed here, i.e., SF-TDDFT/TDA or simply SF-DFT ${ }^{57-61}$ because it is expected to provide a better description of open-shell systems (such as bond-breaking process and biradical) than the traditional Kohn-Sham density functional theory (KS-DFT). ${ }^{57-65}$ In addition, recent work has suggested that the SF-DFT method can give the correct topology of the $S_{0} / S_{1}$ conical intersection, which is quite critical for the current simulation. ${ }^{61,66}$ The BHHLYP functional $(50 \%$ Hartree-Fock $+50 \%$ Becke for exchange and LYP for correlation) was selected in the SF-DFT calculation. ${ }^{67}$ Certainly, the performance of the SF-DFT with the BHHLYP functional should be carefully examined. For validation, the current results at the SF-DFT/BHHLYP (or simply SFBHHLYP) level were compared with previous data ${ }^{26}$ at the DFT/TDDFT and the second-order multiconfigurational perturbation theory (CASPT2) levels. Due to the inclusion of a large fraction of the Hartree-Fock exchange, ${ }^{60,61}$ the SF-DFT calculations with the BHHLYP functional give a good description of the current system, ${ }^{63}$ which includes a CT process. $^{68,69}$ The $6-31 \mathrm{G}^{* *}$ basis set ${ }^{70,71}$ was used in the SFDFT TSH simulation. For comparison, the 6-31G basis set was also employed.

Currently, the analytical nonadiabatic couplings at the SFDFT level still represent a great challenge. ${ }^{66}$ Thus, in the current paper, a more straightforward approach was employed to numerically estimate the nonadiabatic coupling by computing the wave function overlaps ${ }^{72,73}$ of different electronic states at two successive time steps with a small duration. The SF-DFT calculations and the TSH simulation were performed using the US variant of General Atomic and 
Molecular Electronic Structure System (GAMESS-US) ${ }^{67}$ and Newton-X program package, ${ }^{74,75}$ respectively. To connect them, a new interface between Newton-X and GAMESS-US was added to allow the usage of SF-DFT in TSH calculations. The CIOVERALP program in Newton-X, which was originally designed for closed-shell reference, was adapted to calculate the nonadiabatic coupling terms of the open-shell reference of SFDFT.

The Newton equations were integrated using the Velocity Verlet algorithm ${ }^{76-78}$ with a constant time step of $0.5 \mathrm{fs}$ and a maximum simulation time of $150 \mathrm{fs}$. In the initial samplings, random velocities at a constant temperature of $300 \mathrm{~K}$ and a fixed TS geometry were sampled as an initial condition, according to the algorithm proposed by Sellner et al. ${ }^{79}$ Thus, the statistical behavior of the trajectories can be described by the canonical (NVT) ensemble. The zero-point vibrational energy of the TS, including all real vibrational modes $(4.14 \mathrm{eV}$ for $6-31 \mathrm{G}^{* *}$ and $4.17 \mathrm{eV}$ for $6-31 \mathrm{G}$ basis set, respectively), was chosen as the initial kinetic energy.

\section{ASSOCIATED CONTENT}

\section{S Supporting Information}

The $S_{0}$ and $S_{1}$ PES and the Mulliken charge population of $S_{0}$ PES, and the comparison of $S_{0}$ PES calculated at SF-BHHLYP/ 6-31G, CAM-B3LYP/6-31G**, CASPT2/ANO-RCC-VDZP levels; detailed two-dimensional distribution of $\mathrm{C}_{1}-\mathrm{C}_{2}$ and $\mathrm{O}_{3}-\mathrm{O}_{4}$ bond lengths from 10.0 to 150.0 fs at SF-BHHLYP/6$31 \mathrm{G}$ and SF-BHHLYP/6-31G** levels; the results of TSH simulation at SF-BHHLYP/6-31G level; Cartesian coordinates for the Min and TS of FDO $^{-}$optimized by SF-BHHLYP method. This material is available free of charge via the Internet at http://pubs.acs.org.

\section{AUTHOR INFORMATION}

\section{Corresponding Authors}

*E-mail: lanzg@qibebt.ac.cn (Z.-G. L).

*E-mail: yajun.liu@bnu.edu.cn (Y.-J. L).

\section{Notes}

The authors declare no competing financial interest.

\section{ACKNOWLEDGMENTS}

This work was supported by grants from the National Natural Science Foundation of China (Grant Nos. 21273021, 21325312, and 21421003) and the Major State Basic Research Development Programs (Grant No. 2011CB808500). Financial supported from the CAS 100 Talent Project, National Science Foundation of China (Grant Nos. 21103213, 91233106), and Key Lab of Nanodevics and Nanoapplications, CAS (Grant Nos. 14HZ03). The authors also thank the Supercomputing Center, Computer Network Information Center, CAS, national supercomputing center in Shenzhen, and the super computational center of CAS-QIBEBT for providing computational resources.

\section{REFERENCES}

(1) Ando, Y.; Niwa, K.; Yamada, N.; Enomoto, T.; Irie, T.; Kubota, H.; Ohmiya, Y.; Akiyama, H. Firefly Bioluminescence Quantum Yield and Colour Change by $\mathrm{pH}-$ Sensitive Green Emission. Nat. Photonics 2008, 2, 44-47.

(2) Shimomura, O. The Fireflies and Luminous Insects. In Bioluminescence Chemical Principles and Methods; World Scientific Publishing Co. Pte. Ltd.: Singapore, 2006; pp 1-29.
(3) Hirano, T.; Hasumi, Y.; Ohtsuka, K.; Maki, S.; Niwa, H.; Yamaji, M.; Hashizume, D. Spectroscopic Studies of the Light-Color Modulation Mechanism of Firefly (Beetle) Bioluminescence. I. Am. Chem. Soc. 2009, 131, 2385-2396.

(4) Naumov, P.; Ozawa, Y.; Ohkubo, K.; Fukuzumi, S. Structure and Spectroscopy of Oxyluciferin, the Light Emitter of the Firefly Bioluminescence. I. Am. Chem. Soc. 2009, 131, 11590-11605.

(5) Sakai, H.; Wada, N. Theoretical analysis on the absorption spectra of intermediates of firefly luciferin in deoxygenated dimethyl sulfoxide. Luminescence 2008, 23, 90 Abstracts of the 15th International Symposium on Bioluminescence and Chemiluminescence.

(6) Orlova, G.; Goddard, J. D.; Brovko, L. Y. Theoretical Study of the Amazing Firefly Bioluminescence: The Formation and Structures of the Light Emitters. I. Am. Chem. Soc. 2003, 125, 6962-6971.

(7) Nakatani, N.; Hasegawa, J.-Y.; Nakatsuji, H. Red Light in Chemiluminescence and Yellow-Green Light in Bioluminescence: Color-Tuning Mechanism of Firefly, Photinus pyralis, Studied by the Symmetry-Adapted Cluster-Configuration Interaction Method. I. Am. Chem. Soc. 2007, 129, 8756-8765.

(8) Min, C.-G.; Ren, A.-M.; Guo, J.-F.; Zou, L.-Y.; Goddard, J. D.; Sun, C.-C. Theoretical Investigation on the Origin of Yellow-Green Firefly Bioluminescence by Time-Dependent Density Functional Theory. ChemPhysChem 2010, 11, 2199-2204.

(9) Chen, S.-F.; Liu, Y.-J.; Navizet, I.; Ferré, N.; Fang, W.-H.; Lindh, R. Systematic Theoretical Investigation on the Light Emitter of Firefly. I. Chem. Theorv. Comput. 2011, 7, 798-803.

(10) Liu, Y.-J.; Vico, L. D.; Lindh, R. Ab Initio Investigation on the Chemical Origin of the Firefly Bioluminescence. I. Photochem. Photobiol., A: Chem. 2008, 194, 261-267.

(11) McCapra, F.; Beheshti, I.; Burford, A.; Hann, R. A.; Zaklika, K. A. Singlet Excited States from Dioxetan Decomposition. I. Chem. Soc., Chem. Commun. 1977, 944-946.

(12) Zaklika, K. A.; Kissel, T.; Thayer, A. L.; Burns, P. A.; Schaap, A. P. Mechanism of 1,2-Dioxetane Decomposition: The Role of Electron Transfer. Photochem. Photobiol. 1979, 30, 35-44.

(13) Schmidt, S. P.; Schuster, G. B. Dioxetanone Chemiluminescence by the Chemically Initiated Electron Exchange Pathway. Efficient Generation of Excited Singlet States. I. Am. Chem. Soc. 1978, 100, 1966-1968.

(14) Koo, J.-Y.; Schmidt, S. P.; Schuster, G. B. Bioluminescence of the Firefly: Key Steps in the Formation of the Electronically Excited State for Model Systems. Proc. Natl. Acad. Sci. U. S. A. 1978, 75, 3033.

(15) Bronstein, I.; McGrath, P. Chemiluminescence Lights Up. Nature 1989, 338, 599-600.

(16) Matsumoto, M.; Suganuma, H.; Katao, Y.; Mutoh, H. Thermal Stability and Chemiluminescence of 3-Alkoxy-3-aryl-4,4-diisopropyl1,2-dioxetanes. I. Chem. Soc., Chem. Commun. 1995, 0, 431-432.

(17) Koo, J.-Y.; Schuster, G. B. Chemically Initiated Electron Exchange Luminescence. A New Chemiluminescent Reaction Path for Organic Peroxides. I. Am. Chem. Soc. 1977, 99, 6107-6109.

(18) Schuster, G. B. Chemiluminescence of Organic Peroxides. Conversion of Ground-State Reactants to Excited-State Products by the Chemically Initiated Electron-Exchange Luminescence Mechanism. Acc. Chem. Res. 1979, 12, 366-373.

(19) Isobe, H.; Takano, Y.; Okumura, M.; Kuramitsu, S.; Yamaguchi, $\mathrm{K}$. Mechanistic Insights in Charge-Transfer-Induced Luminescence of 1, 2-Dioxetanones with a Substituent of Low Oxidation Potential. I. Am. Chem. Soc. 2005, 127, 8667-8679.

(20) Isobe, H.; Yamanaka, S.; Kuramitsu, S.; Yamaguchi, K. Regulation Mechanism of Spin-Orbit Coupling in Charge-TransferInduced Luminescence of Imidazopyrazinone Derivatives. I. Am. Chem. Soc. 2008, 130, 132-149.

(21) Isobe, H.; Takano, Y.; Yoshika, Y.; Yamaguchi, K. Theoretical Study on Decomposition of Dioxetanone. Bunshi Kozo Sogo Toronkai Koen Yoshishu 1999, 322.

(22) Matsumoto, M.; Suzuki, H.; Sano, Y.; Watanabe, N.; Ijuin, H. K. Rotamer-Dependent Chemiluminescence in the Intramolecular 
Charge-Transfer-Induced Decomposition of Bicyclic Dioxetanes Bearing a Hydroxyaryl Group. Tetrahedron Lett. 2008, 49, 5372-5375.

(23) Matsumoto, M.; Kakuno, F.; Kikkawa, A.; Hoshiya, N.; Watanabe, N.; Ijuin, H. K. Alkaline Metal Ion-Enhanced Chemiluminescence of Bicyclic Dioxetanes Bearing a Hydroxyaryl Group with an 'Even' Substitution Pattern. Tetrahedron Lett. 2009, 50, 23372341.

(24) Tanimura, M.; Watanabe, N.; Ijuin, H. K.; Matsumoto, M. Thermodynamic Aspects of Thermal Decomposition and ChargeTransfer-Induced Chemiluminescent Decomposition for Bicyclic Dioxetanes Bearing a 4-(Benzothiazol-2-yl)-3-hydroxyphenyl Moiety. I. Org. Chem. 2010, 75, 3678-3684.

(25) Watanabe, N.; Kikuchi, M.; Maniwa, Y.; Ijuin, H. K.; Matsumoto, M. Synthesis of Sulfanyl-, Sulfinyl-, and SulfonylSubstituted Bicyclic Dioxetanes and Their Base-Induced Chemiluminescence. I. Org. Chem. 2010, 75, 879-884.

(26) Yue, L.; Liu, Y.-J.; Fang, W.-H. Mechanistic Insight into the Chemiluminescent Decomposition of Firefly Dioxetanone. I. Am. Chem. Soc. 2012, 134, 11632-11639.

(27) Farahani, P.; Roca-Sanjuán, D.; Zapata, F.; Lindh, R. Revisiting the Nonadiabatic Process in 1,2-Dioxetane. I. Chem. Theorv. Comput. 2013, 9, 5404-5411.

(28) Vico, L. D.; Liu, Y.-J.; Krogh, J. W.; Lindh, R. Chemiluminescence of 1,2-Dioxetane. Reaction Mechanism Uncovered. I. Phys. Chem. A 2007, 111, 8013-8019.

(29) Liu, F.; Liu, Y.-J.; Vico, L. D.; Lindh, R. A CASSCF/CASPT2 Approach to the Decomposition of Thiazole-Substituted Dioxetanone: Substitution Effects and Charge-Transfer Induced Electron Excitation. Chem. Phvs. Lett. 2009, 484, 69-75.

(30) Liu, F.; Liu, Y.-J.; Vico, L. D.; Lindh, R. Theoretical Study of the Chemiluminescent Decomposition of Dioxetanone. I. Am. Chem. Soc. 2009, 131, 6181-6188.

(31) Wada, N.; Sakai, H. Decomposition Reaction of Dioxetanone in Firefly Bioluminescence by Computer Experiment. I. Biol. Phys. 2005, 31, 403-412.

(32) Yue, L.; Roca-Sanjuán, D.; Lindh, R; Ferré, N.; Liu, Y.-J. Can the Closed-Shell DFT Methods Describe the Thermolysis of 1,2Dioxetanone? I. Chem. Theorv. Comput. 2012, 8, 4359-4363.

(33) Yue, L.; Liu, Y.-J. Mechanism of AMPPD Chemiluminescence in a Different Voice. L. Chem. Theory. Comput. 2013, 9, 2300-2312.

(34) White, E. H.; Rapaport, E.; Seliger, H. H.; Hopkins, T. A. The Chemi- and Bioluminescence of Firefly Luciferin: An Efficient Chemical Production of Electronically Excited States. Bioorg. Chem. 1971, 1, 92-122.

(35) Schaap, A. P.; Gagnon, S. D. Chemiluminescence from a Phenoxide-Substituted 1, 2-Dioxetane: A Model for Firefly Bioluminescence. I. Am. Chem. Soc. 1982, 104, 3504-3506.

(36) Chung, L. W.; Hayashi, S.; Lundberg, M.; Nakatsu, T.; Kato, H.; Morokuma, K. Mechanism of Efficient Firefly Bioluminescence via Adiabatic Transition State and Seam of Sloped Conical Intersection. $L$. Am. Chem. Soc. 2008, 130, 12880-12881.

(37) Kricka, L. J.; Thorpe, G. H. G. Chemiluminescent and Bioluminescent Methods in Analytical Chemistry. A Review. Analyst 1983, 108, 1274-1296.

(38) Voyta, J. C.; Edwards, B.; Bronstein, I. Scientific program. American Association for Clinical Chemistry, 40th National Meeting. July 24-28, 1988, New Orleans, Louisiana. Abstracts. Clin. Chem. 1988, 34, 1157.

(39) Bronstein, I.; Edwards, B.; Voyta, J. C. 1,2-dioxetanes: Novel Chemiluminescent Enzyme Substrates. Applications to Immunoassays. I. Biolumin. Chemilumin. 1989, 4, 99-111.

(40) Thorpe, G. H.; Bronstein, I.; Kricka, L. J.; Edwards, B.; Voyta, J. C. Chemiluminescent Enzyme Immunoassay of Alpha-Fetoprotein Based on an Adamantyl Dioxetane Phenyl Phosphate Substrate. Clin. Chem. 1989, 35, 2319-2339.

(41) Bronstein, I.; Martin, C. S.; Fortin, J. J.; Olesen, C. E.; Voyta, J. C. Chemiluminescence: Sensitive Detection Technology for Reporter Gene Assays. Clin. Chem. 1996, 42, 1542-1547.
(42) Dodeigne, C.; Thunus, L.; Lejeune, R. Chemiluminescence as Diagnostic Tool. A Review. Talanta 2000, 51, 415-439.

(43) Kricka, L. J. Clinical Applications of Chemiluminescence. Anal. Chim. Acta 2003, 500, 279-286.

(44) El-Sayed, M. A. Spin-Orbit Coupling and the Radiationless Processes in Nitrogen Heterocyclics. I. Chem. Phys. 1963, 38, 28342838.

(45) White, E. H.; Steinmetz, M. G.; Miano, J. D.; Wildes, P. D.; Morland, R. Chemi- and Bioluminescence of Firefly Luciferin. $\underline{\text { L. Am. }}$. Chem. Soc. 1980, 102, 3199-3208.

(46) Tully, J. C. Mixed Quantum-Classical Dynamics. Faradav Discuss. 1998, 110, 407-419.

(47) Tully, J. C. Molecular Dynamics with Electronic Transitions. I. Chem. Phys. 1990, 93, 1061-1071.

(48) Shenvi, N.; Subotnik, J. E.; Yang, W. Phase-Corrected Surface Hopping: Correcting the Phase Evolution of the Electronic Wavefunction. I. Chem. Phvs. 2011, 135, 024101.

(49) Granucci, G.; Persico, M. Critical Appraisal of the Fewest Switches Algorithm for Surface Hopping. I. Chem. Phys. 2007, 126, 134114.

(50) Granucci, G.; Persico, M.; Zoccante, A. Including Quantum Decoherence in Surface Hopping. I. Chem. Phvs. 2010, 133, 134111.

(51) Krylov, A. I. Spin-Flip Configuration Interaction: An Electronic Structure Model That Is Both Variational and Size-Consistent. Chem. Phvs. Lett. 2001, 350, 522-530.

(52) Krylov, A. I. Size-Consistent Wave Functions for BondBreaking: The Equation-of-Motion Spin-Flip Model. Chem. Phvs. Lett. 2001, 338, 375-384.

(53) Krylov, A. I.; Sherrill, C. D. Perturbative Corrections to the Equation-of-Motion Spin-Flip Self-Consistent Field Model: Application to Bond-Breaking and Equilibrium Properties of Diradicals. I. Chem. Phvs. 2002, 116, 3194-3203.

(54) Casanova, D.; Head-Gordon, M. The Spin-Flip Extended Single Excitation Configuration Interaction Method. I. Chem. Phys. 2008, 129, 064104.

(55) Hirata, S.; Head-Gordon, M. Time-Dependent Density Functional Theory within the Tamm-Dancoff Approximation. Chem. Phvs. Lett. 1999, 314, 291-299.

(56) Liu, F.; Gan, Z.; Shao, Y.; Hsu, C.-P.; Dreuw, A.; Head-Gordon, M.; Miller, B. T.; Brooks, B. R.; Yu, J.-G.; Furlani, T. R.; Kong, J. A Parallel Implementation of the Analytic Nuclear Gradient for TimeDependent Density Functional Theory within the Tamm-Dancoff Approximation. Mol. Phvs. 2010, 108, 2791-2800.

(57) Huix-Rotllant, M.; Natarajan, B.; Ipatov, A.; Muhavini Wawire, C.; Deutsch, T.; Casida, M. E. Assessment of Noncollinear Spin-Flip Tamm-Dancoff Approximation Time-Dependent Density-Functional Theory for the Photochemical Ring-Opening of Oxirane. Phvs. Chem. Chem. Phvs. 2010, 12, 12811-12825.

(58) Yang, K.; Peverati, R.; Truhlar, D. G.; Valero, R. Density Functional Study of Multiplicity-Changing Valence and Rydberg Excitations of p-Block Elements: Delta Self-Consistent Field, Collinear Spin-Flip Time-Dependent Density Functional Theory (DFT), and Conventional Time-Dependent DFT. I. Chem. Phys. 2011, 135, 044118.

(59) Seth, M.; Mazur, G.; Ziegler, T. Time-Dependent Density Functional Theory Gradients in the Amsterdam Density Functional Package: Geometry Optimizations of Spin-Flip Excitations. Theor. Chem. Acc. 2011, 129, 331-342.

(60) Bernard, Y. A.; Shao, Y.; Krylov, A. I. General Formulation of Spin-Flip Time-Dependent Density Functional Theory Using Noncollinear Kernels: Theory, Implementation, and Benchmarks. I. Chem. Phvs. 2012, 136--.

(61) Harabuchi, Y.; Maeda, S.; Taketsugu, T.; Minezawa, N.; Morokuma, K. Automated Search for Minimum Energy Conical Intersection Geometries between the Lowest Two Singlet States $\mathrm{S}_{0} /$ $S_{1}$-MECIs by the Spin-Flip TDDFT Method. I. Chem. Theorv. Comput. 2013, 9, 4116-4123. 
(62) Slipchenko, L. V.; Krylov, A. I. Singlet-Triplet Gaps in Diradicals by the Spin-Flip Approach: A Benchmark Study. I. Chem. Phvs. 2002, 117, 4694-4708.

(63) Shao, Y.; Head-Gordon, M.; Krylov, A. I. The Spin-Flip Approach within Time-Dependent Density Functional Theory: Theory and Applications to Diradicals. I. Chem. Phvs. 2003, 118, 4807-4818.

(64) Rinkevicius, Z.; Ågren, H. Spin-Flip Time Dependent Density Functional Theory for Singlet-Triplet Splittings in $\sigma, \sigma$-Biradicals. Chem. Phvs. Lett. 2010, 491, 132-135.

(65) Rinkevicius, Z.; Vahtras, O.; Agren, H. Spin-Flip Time Dependent Density Functional Theory Applied to Excited States with Single, Double, or Mixed Electron Excitation Character. $\underline{\text { I. Chem. }}$ Phvs. 2010, 133, 114104.

(66) Minezawa, N.; Gordon, M. S. Optimizing Conical Intersections by Spin-Flip Density Functional Theory: Application to Ethylene. $L$. Phvs. Chem. A 2009, 113, 12749-12753.

(67) Schmidt, M. W.; Baldridge, K. K.; Boatz, J. A.; Elbert, S. T.; Gordon, M. S.; Jensen, J. H.; Koseki, S.; Matsunaga, N.; Nguyen, K. A.; Su, S.; Windus, T. L.; Dupuis, M.; Montgomery, J. A. General Atomic and Molecular Electronic Structure System. L. Comput. Chem. 1993, 14, 1347-1363.

(68) Dreuw, A.; Head-Gordon, M. Failure of Time-Dependent Density Functional Theory for Long-Range Charge-Transfer Excited States: The Zincbacteriochlorin-Bacteriochlorin and Bacteriochlorophyll-Spheroidene Complexes. L. Am. Chem. Soc. 2004, 126, 40074016.

(69) Vydrov, O. A.; Heyd, J.; Krukau, A. V.; Scuseria, G. E. Importance of Short-Range versus Long-Range Hartree-Fock Exchange for the Performance of Hybrid Density Functionals. I. Chem. Phvs. 2006, 125, 074106-074114.

(70) Hehre, W. J.; Ditchfield, R.; Pople, J. A. Self-Consistent Molecular Orbital Methods. XII. Further Extensions of Gaussian-Type Basis Sets for Use in Molecular Orbital Studies of Organic Molecules. I. Chem. Phys. 1972, 56, 2257-2261.

(71) Hariharan, P. C.; Pople, J. A. The Influence of Polarization Functions on Molecular Orbital Hydrogenation Energies. Theor. Chem. Acc. 1973, 28, 213-222.

(72) Pittner, J.; Lischka, H.; Barbatti, M. Optimization of Mixed Quantum-Classical Dynamics: Time-Derivative Coupling Terms and Selected Couplings. Chem. Phvs. 2009, 356, 147-152.

(73) Werner, U.; Mitrić, R.; Suzuki, T.; Bonačić-Koutecký, V. Nonadiabatic Dynamics within the Time Dependent Density Functional Theory: Ultrafast Photodynamics in Pyrazine. Chem. Phvs. 2008, 349, 319-324.

(74) Barbatti, M.; Granucci, G.; Persico, M.; Ruckenbauer, M.; Vazdar, M.; Eckert-Maksić, M.; Lischka, H. The On-the-Fly SurfaceHopping Program System Newton-X: Application to Ab Initio Simulation of the Nonadiabatic Photodynamics of Benchmark Systems. I. Photochem. Photobiol., A: Chem. 2007, 190, 228-240.

(75) Barbatti, M.; Granucci, G.; Ruckenbauer, M.; Pittner, J.; Persico, M.; Lischka, H. NEWTON-X: A Package for Newtonian Dynamics Close to the Crossing Seam, version 1.3, 2012, www.newtonx.org.

(76) Verlet, L. Computer "Experiments" on Classical Fluids. I. Thermodynamical Properties of Lennard-Jones Molecules. Phvs. Rev. 1967, 159, 98-103.

(77) Verlet, L. Computer "Experiments" on Classical Fluids. II. Equilibrium Correlation Functions. Phvs. Rev. 1968, 165, 201-214.

(78) Swope, W. C.; Andersen, H. C.; Berens, P. H.; Wilson, K. R. A Computer Simulation Method for the Calculation of Equilibrium Constants for the Formation of Physical Clusters of Molecules: Application to Small Water Clusters. I. Chem. Phys. 1982, 76, 637649.

(79) Sellner, B.; Barbatti, M.; Lischka, H. Dynamics Starting at a Conical Intersection: Application to the Photochemistry of Pyrrole. $L$. Chem. Phvs. 2009, 131, 024312. 